10.2478/gb-2021-0006

sciendo

\title{
Archetypen und das kollektive Unbewusste in Hermann Hesses Demian (1919) und in Urtrost (1924) von Áron Tamási. Ein Vergleich ${ }^{1}$
} Réka JAKABHÁZI

Lekt. Dr., Babeș-Bolyai-Universität Klausenburg;

E-Mail: jakabhazireka@gmail.com

\begin{abstract}
The aim of the present article is to provide a comparative analysis between two important works of German and Hungarian literature on the background of the theory of Carl Gustav Jung's theory of archetypes. Both works (the novel Demian by Hermann Hesse and the drama Erstwhile Solace [Ösvigasztalás] by Áron Tamási) approach the theme of the search for identity as well as for the absolute and the divinity, with the focus on the archetypes of the collective unconscious. Motifs such as androgyny, shadow or dream, the issue of polarity and unity form common points of contact between the two analysed literary works.
\end{abstract}

Keywords: collective unconscious, archetypes, myth, comparative analysis, Hermann Hesse, Áron Tamási.

Ziel des vorliegenden Beitrags ist es, einen Vergleich zwischen zwei bedeutenden Werken der deutschen und der ungarischen Literatur aus der ersten Hälfte des 20. Jahrhunderts auf dem Hintergrund der Archetypenlehre von Carl Gustav Jung anzustellen. Beide Texte, der Roman Demian ${ }^{2}$ von Hermann Hesse

${ }^{1}$ Die Forschung wurde vom Domus-Stipendienprogramm der Ungarischen Akademie der Wissenschaften gefördert.

2 Hesse, Hermann: Demian. Die Geschichte von Emil Sinclairs Jugend. Berlin: Fischer 1920. 
und das Drama Urtrost (Ősvigasztalás) $)^{3}$ von Áron Tamási, thematisieren die Suche des Einzelnen nach Identität und Daseinsberechtigung, wobei der Fokus wiederholt auf das Wesen und die Bedeutung des kollektiven Unbewussten gelegt wird. Motive wie der Schatten, der Urgott, oder der Traum, das Problem der Polarität und Einheit bilden gemeinsame Anknüpfungspunkte zwischen den behandelten Werken.

Da eine ausführliche Behandlung der Jungschen Theorie des kollektiven Unbewussten den Rahmen dieses Beitrags sprengen würde, werde ich im Folgenden nur auf die für unsere Untersuchung relevanten Aspekte des Themas eingehen.

In seinem Buch Die Archetypen und das kollektive Unbewusste $^{4}$ entwickelt Carl Gustav Jung seine Theorie von einem kollektiven Unbewussten, worunter hier eine Art Menschheitsgedächtnis verstanden wird. Dadurch grenzt er sich von Sigmund Freuds Vorstellungen eines individuellen Unbewussten ab, bzw. er erweitert die Perspektive der freudschen Psychoanalyse: Während Freud im Reifeprozess des Menschen dem Sexualtrieb bzw. seiner Unterdrückung eine gewichtige Rolle zukommen ließ, rückte Jung in seinen Ausführungen den Einfluss der Verbindung zu den Urahnen in den Fokus: „Das Kollektive Unbewusste ist die gewaltige geistige Erbmasse der Menschheitsentwicklung; wiedergeboreninjederindividuellenHirnstruktur. “5

In seiner Theorie weist Jung darauf hin, dass es wiederkehrende Symbole gibt, die auch in Märchen und Mythen zu finden sind; diese weisen über die Grenzen der Kulturen bzw. Sprachen hinaus und sind allen Menschen zu eigen. Darin sieht

3 Tamási, Áron: Ösvigasztalás. In: Tiszatáj 27 (1973), H.5, S. 6-25, bzw. Tiszatáj 27 (1973), H.6, S. 23-48.

4 Jung, Carl Gustav: Die Archetypen und das kollektive Unbewußte. [Gesammelte Werke Bd. 9/1], Olten: Walter 1976.

5 Jung, Carl Gustav: Die Struktur der Seele. In: Ders.: Die Dynamik des Unbewussten. [Gesammelte Werke, Bd. 8], Ostfildern: Patmos 1995, S. 181. 
er den Beweis für eine allen Menschen gemeinsame Instanz, für das kollektive Unbewusste.

Bereits Sigmund Freud richtete das Augenmerk auf die Wichtigkeit der symbolischen Bilder bzw. Träume, die vergleichbar sind mit den Bildern der Mythen, der Riten und den Elementen der traditionellen Folklore. Nach Freud entspringen Traum und Mythos aus einem gemeinsamen Stamm, ihr Ursprung ist jedoch unbekannt. Freud spricht dabei über ,,archaische Erbschaft" und ist der Ansicht, dass diese Elemente seit den Urzeiten in der menschlichen Psyche des Individuums existieren. Jung weist darauf hin, dass diese, von Freud als ,archaische Erbschaft" bezeichneten Motive, ererbte Formen der menschlichen Psyche zu sein scheinen und universell sind. Sie können also nicht mit dem Unbewussten des Individuums verbunden, sondern müssen dem kollektiven Unbewussten der Menschheit zugeordnet werden. Für die Benennung dieser, im kollektiven Unbewussten auf natürliche Weise präsenten Bilder führt Jung den Begriff Archetyp ein.

Die Archetypen versteht Jung als vorgefertigte Grundstrukturen der menschlichen Psyche; Symbole oder Urbilder, die allen Menschen gemeinsam und tief verankert im kollektiven Unbewussten sind, und von hier aus unser Selbstverständnis, das Wahrnehmen des Ichs und der Welt bzw. unser Handeln bestimmen. Diese archetypischen Leitbilder sind also präexistente Schablonen, die die wesentlichen Wirkkräfte des Lebens verständlich machen.

Die Archetypenlehre von Carl Gustav Jung übte einen weltweit nachhaltigen Einfluss nicht nur auf die Psychologie und Medizin, sondern auch auf Literatur, Philosophie und Kunst aus. Einer der ersten deutschen Schriftsteller, die sich in ihren Werken schwerpunktmäßig mit dem Thema des individuellen und kollektiven Unbewussten, bzw. mit der führenden Kraft der Archetypen als Wegweiser auf dem Individuationsweg der Protagonisten beschäftigt haben, war Hermann Hesse. Er kam 
persönlich mit den Lehren von Jung in Kontakt, als er nach einem Nervenzusammenbruch den Psychoanalytiker Josef Bernhard Lang, einen Schüler von C.G. Jung, aufsuchte und sich von ihm behandeln ließ. Der Roman Demian kann als direktes Resultat ihrer Gespräche bezeichnet werden, worauf auch Hugo Ball, Hesses erster Biograf hinweist. ${ }^{6}$ Hesse verewigte seinen Therapeuten in der Figur des Organisten Pistorius.

Das Hauptthema des Romans Demian bildet der Individuationsweg eines Knaben, der im Laufe der Handlung die auf Polarität beruhende Welt hinter sich lässt und die Einheit mit sich und der Welt erlangt. Dieser Individuationsweg, bzw. die Entwicklung der Identität von Emil Sinclair, ist stark von vererbten Mythen und Symbolen geprägt: Die Suche nach der eigenen Identität stimmt mit der Suche nach Glauben und Religiosität überein. Doch diese Religiosität weist über das Christentum hinaus und zeigt Spuren des Gnostizismus und Taoismus. Am Anfang der Romanhandlung lernen wir Emil in seiner streng christlich-religiösen Umgebung kennen, ein Kind, das schon im frühesten Kindesalter die parallele Existenz zweier Welten in seinem Leben wahrnimmt: Eine warme, helle Welt und die ihr gegenübergestellte dunkle, böse Welt:

Die eine Welt war das Vaterhaus [...]. Zu dieser Welt gehörte milder Glanz, Klarheit und Sauberkeit, hier waren sanfte freundliche Reden, gewaschene Hände, reine Kleider, gute Sitten daheim [...]. In dieser Welt gab es [...] Pflicht und Schuld, schlechtes Gewissen und Beichte, Verzeihung und gute Vorsätze, Liebe und Verehrung, Bibelwort und Weisheit. Zu dieser Welt mußte man sich halten, damit das Leben klar und reinlich, schön und geordnet sei. Die andere Welt indessen begann schon mitten in unserem eigenen Hause und war völlig anders, roch anders, sprach anders, versprach und forderte anderes. In dieser zweiten Welt gab es Dienstmägde und Handwerksburschen, Geistergeschichten und Skandalgerüchte [...], Sachen wie Schlachthaus und Gefängnis, Betrunkene und keifende

${ }^{6}$ Ball, Hugo: Hermann Hesse: Sein Leben und sein Werk. Oberursel: Gröls Verlag [Edition Werke der Weltliteratur] 2019, S. 105. 
Weiber, gebärende Kühe, gestürzte Pferde, Erzählungen von Einbrüchen, Totschlägen, Selbstmorden.

Der Individuationsweg bzw. die Suche nach dem eigenen Selbst des jungen Sinclairs vollzieht sich mit Hilfe mythologischer und traumhafter Motive, aber vor allem durch die Führung durch den älteren Freund Demian, eine Art Seelenführer. Als erste Station der Selbstkenntnis lernt er die „Schattenseiten“ der eigenen, als behütet wahrgenommenen Welt kennen, und später sieht er sich mit den nicht so konventionellen Aspekten der biblischen Lehre konfrontiert. Sein Freund Demian präsentiert ihm eine ganz andere Version und Interpretation der biblischen Geschichte von Kain und Abel: Kain wird als Repräsentant der Herrenmoral und Abel als Repräsentant der Sklavenmoral in nietzscheanischem Sinne gedeutet. Kain wird hier als überlegen präsentiert, einer, der den anderen Menschen aufgrund seines eigenen Willens und seines kühnen Charakters unheimlich und befremdlich erscheint. Nach dem Brudermord wird er von allen gefürchtet und erhält von Gott ein Zeichen auf die Stirn. Demian deutet dieses Kainszeichen nicht als Schandmal, sondern als Auszeichnung der geheimnisvoll Überlegenen. Den Erklärungen Demians zufolge wird dieses Kainszeichen von den Erwachten getragen und als Symbol für ein hohes Maß an Individuation gedeutet. Später trifft Sinclair auf Pistorius, der mit ihm lange Gespräche über Gott und Glauben führt und ihm über Abraxas erzählt, die Gottheit, die die absolute Einheit der Gegensätze verkörpert:

Lieber Sinclair, unser Gott heißt Abraxas, und er ist Gott und ist Satan, er hat die lichte und die dunkle Welt in sich. Abraxas hat gegen keinen Ihrer Gedanken, gegen keinen Ihrer Träume etwas einzuwenden.

DerEinfluss der jungschen Theorie des kollektiven Unbewussten widerspiegelt sich in den folgenden Zeilen:

Wir ziehen die Grenzen unserer Persönlichkeit immer viel zu eng! Wir rechnen zu unserer Person immer bloß das, was wir als 
individuell unterschieden, als abweichend erkennen. Wir bestehen aber aus dem ganzen Bestand der Welt, jeder von uns [...]. Alle Götter und Teufel, die je gewesen sind, sei es bei Griechen und Chinesen oder bei Zulu-Kaffern, alle sind mit in uns [...]. Wenn die Menschheit ausstürbe bis auf ein einziges, halbwegs begabtes Kind, das keinerlei Unterricht genossen hat [...], es würde Götter, Dämonen, Paradiese, Gebote und Verbote, Alte und Neue Testamente, alles würde es wieder produzieren können.

Die polare Welt als Raum der Erfahrung und der Imagination bzw. das Problem der Religiosität erscheint als Themenschwerpunkt auch im Drama Urtrost [Ösvigasztalás] von Áron Tamási.

Die epischen Werke des aus einer Szekler Bauernfamilie stammenden Tamási zeigen bereits in den ersten Schaffensjahren eine bemerkenswerte Öffnung zum Volkshaften und Volkstümlichen, was mit einer archaisch-mythischen Weltsicht und Religiosität verknüpft in Erscheinung tritt. Sein erster Band Lélekindulás [Seelenaufbruch] besteht aus mehreren Novellen, die von den Szekler Volksballaden und Volksliedern inspiriert wurden. Die Themen dieser epischen Texte entstammen bevorzugt dem Alltag der Szekler, in einer Umgebung, wo christliche Religiosität kombiniert mit spiritualistischen Auffassungen und heidnischer Mystik das Leben der Protagonisten bestimmen. Diese Welt wird in expressiver Anschaulichkeit und mit Hilfe vieler Symbole dargestellt, wobei auch die Sprache diese archaisch-mystische Atmosphäre überzeugend wiedergibt: Regionalismen, Archaismen sowie eine ausdrucksstarke und ungewohnte Bildsprache sind für diese Texte charakteristisch.

Als Hauptquelle des dramatischen Werkes Urtrost dient ebenfalls die archaische Spiritualität sowie die althergebrachte und im kollektiven Gedächtnis der Szekler immer noch sehr lebendige Geschichte dieser Volksgruppe. Trotz der Tatsache, dass die Handlung nach den Anweisungen des Autors ,,im späten 19. Jahrhundert im Szeklerland stattfindet, und führt sogar das zwanzigste Jahrhundert ein", steht im Mittelpunkt des 
Werkes nicht nur eine konkrete Geschichte mit konkreten Figuren, in einer bestimmten Zeit und an einem bestimmten Ort, sondern sie führt uns in die Atmosphäre einer vergessenen Welt, deren Erinnerungen unserer zivilisierten Gesellschaft unterworfen wurden.

Im Drama Urtrost können mehrere Aspekte identifiziert werden, die im Hinblick auf die Theorie des kollektiven Unbewussten bzw. auf die Archetypenlehre von C.G. Jung gedeutet werden können. Die dramatische Situation entsteht durch den ungewöhnlichen letzten Willen des sterbenden Ambrus Csorja: Sein jüngerer Bruder, Ádám, soll seine Leiche auf der Berglichtung im Feuer verbrennen, und damit ein uraltes Opfer für den „Urgott" vollbringen. Ádám hält sein Versprechen, sammelt die Asche seines Bruders in einem Topf und mischt sein eigenes Blut hinein: Er schneidet sich in den Unterarm, um durch sein Blutopfer nicht nur seinem verstorbenen Bruder die gebührende Ehre zu erweisen, sondern dadurch auch den „Urgott" zu würdigen. Als diese Tat ans Tageslicht kommt, wird Ádám Csorja wegen Brudermordes vor Gericht gestellt, und obwohl seine Unschuld aufgrund von Zeugenaussagen nachgewiesen wird, wird er wegen „Verstoßes gegen gesetzliche Vorschriften und Formgebote“"zu zehn Monaten Gefängnis verurteilt. Mithilfe des Gendarmen Bence Gálfi entkommt er aus der Haft, doch als sie feststellen, dass sie beide in dieselbe Jula Kispál verliebt sind, erschießt Gálfi (der sich selbst als „Seelenbruder“ des Protagonisten betrachtet) Csorja. Das biblische Motiv des Brudermordes, die Geschichte von Kain und Abel, ist in diesem Werk ebenfalls stark präsent.

Auch dem Kainszeichen - obwohl dies so nicht angesprochen wird - wird eine symbolische Bedeutung zugesprochen, ähnlich wie im Roman von Hermann Hesse. Ádám und Ambrus Csorja tragen dieses Zeichen - wie Demian, seine Mutter und Sinclair -, da sie Außenseiter sind, die abseits von der zivilisierten Gesellschaft und vom Tempo der immer rascher werdenden 
„neuen“ Welt auf dem Berg nach eigenen Gesetzen leben, die von uralten Traditionen definiert sind: Sie ehren sowohl die Erinnerung der „alten Götter“, (Feuer, Wasser, Mond, Sonne, Erde) unterwerfen sich aber stillschweigend auch den Regeln der katholischen Kirche.

Einen ersten gemeinsamen Punkt zwischen den beiden zu behandelnden Werken, Demian und Urtrost, bildet also die Offenheit gegenüber der (Re)interpretation der Mythen. Im Roman von Hesse orientiert sich vor allem der zweite Teil an den Mythen, wo die Handlung vom Auftreten von Träumen und zahlreichen Symbolen geprägt ist. Eine ausdruckskräftige und konkrete Erscheinung des Mythischen macht sich in der Figur des Abraxas bemerkbar. Dieser wird im Werk sowohl als Gottheit, als höchstes Symbol des Urwesens, als auch als Mythos behandelt und vereint in sich das Göttliche und das Teuflische zugleich, das Gute und das Böse, die Stärke und die Weichheit, er ist ein Symbol der ultimativen Einheit. Dadurch, dass Emil Wesen und Botschaft dieser Göttlichkeit erkennt, versteht er, dass er vom Polaritätsdenken endgültig Abschied nehmen sollte. Ádám Csorja verehrt ebenfalls eine Art Abraxas, auch wenn sein Gott nicht diesen Namen trägt: denn er ist Schöpfer und Zerstörer, Urmutter und Urvater, Feuer und Erde, Licht und Dunkel - Ursprung und Grund aller Dinge, Archetyp der Einheit: der Urgott. Wie der Schriftführer im Gerichtsaal über Ádám aussagt: ,,er hat einen anderen Gott als wir“".

Was bei Hesse Pistorius im Zusammenhang mit der Gottheit Abraxas äußert: „Wenn wir einen Menschen hassen, so hassen wir in seinem Bild etwas, was in uns selber sitzt", dasselbe äußert Ádám Csorja im Drama von Tamási:

Ich empfinde den Menschen als meinen Bruder, und wenn dieser mich verurteilt, so verurteilt er sich selbst. Wenn der Gerichthof und alle Menschen dieser neuen Welt an den Gott glauben, der die Welt erschaffen hat, dessen Zeichen die heilige Sonne und dessen Seele

7 ,külön Istent tartott““. [Übersetzung der Verfasserin]. 
das Feuer ist, der uns im Duft der Blume tröstet und im Sturm seine Wut zeigt, wenn die Stadt an diesen Gott glaubt, dann ist dieser auch mein Urgott, der uns als Brüder erschaffen hat. Wenn dies der Christus ist, über den die Heilige Schrift spricht, und ihn den einen Sohn des wahren Gottes nennt, dann kenne und segne ich ihn. ... Weil ich selber der Sohn der alten Großen bin; derjenige, der sich absichtlich aus dieser verdorbenen neuen Welt herausgerissen hat.

Heidnische Symbolik und archaisch-mythische Traditionen und Rituale, die bei den Szeklern bis ins 20. Jahrhundert eine große Wirkung hatten, vermischen sich mit den nach der christlichen Glaubenslehre durchgeführten Riten der Kirche. Die Verschmelzung des alten und neuen Glaubens, das gleichzeitige Auftreten der Mystik der heidnischen Urkräfte und dem christlichen Glaubensbekenntnis erzeugen in beiden Werken eine Spannung, die durch eine Katastrophe aufgelöst wird: Am Ende von Hesses Roman bricht der zerstörende Krieg aus, der Demians Tod mit sich bringt (dessen Ideal jedoch ins Sinclairs Selbst integriert wird), während Urtrost mit dem gewaltsamen Tod des Protagonisten schließt.

Das Erkennen der Einheit der Gegensätze, bzw. die Identifikation und Integration des Schattens im Sinne von Jung, helfen den Protagonisten der beiden Werke dabei, ihre eigene Identität zu erkennen. Bereits am Anfang der Romanhandlung kann man in Demian die Anwesenheit bzw. Rolle des Mythischen und Mysteriösen im Individuationsprozess in der Luft spüren. Ähnlich ist es bei Tamási der Fall: Bereits in den ersten Zeilen des Dramas erscheinen einige kollektive, geschichtsmythologische Symbole, welche uns zu Carl Gustav Jung und seiner Archetypenlehre zurückführen und die die Atmosphäre des Mythischen, Uralten und Ewig-Andauernden erschaffen.

Die Gestalten im Roman von Hermann Hesse sind alle eher Archetypen als Charaktere, sie alle stellen ein Konzept dar. Demian und Pistorius sind als Archetypen des Seelenführers zu verstehen: Der eine taucht als Retter auf und befreit Emil aus 
dem Unwissen, der andere führt die Arbeit weiter, als ein Freund, bis der Retter zurückkehrt. Der Schatten im Sinne von Jung erscheint hier in der Gestalt von Kromer, der die dunkle Welt für Emil verkörpert. Nach Jung ist die Integration (des Schattens) ein Prozess, bei dem die verborgenen Teile des Selbst ins Bewusstsein gebracht werden. Der Begriff „Schatten“ repräsentiert demnach die unterdrückten oder geleugneten, verweigerten Teile des Selbst. Als Metapher steht er für das Unbewusste. So steht Franz Kromer im Roman symbolisch für die dunkle Seite der jugendlichen Seele, er steht hier für die Personifizierung des Bösen.

Die beiden Frauenfiguren des Romans (Frau Eva und Beatrice) stellen die Anima dar. Die Anima, als Archetyp, der die unbewusste weibliche Seite im Mann darstellt, entfaltet, wie Jung betont, ,von sich aus günstige und ungünstige, helle und dunkle, gute und böse Wirkungen" ${ }^{8}$ Nach Jung zeigt diese gegengeschlechtliche Manifestation im Unbewussten des Mannes „tief eingeprägte Wirkfaktoren der Seele“, die aus dem kollektiven Unbewussten entspringen, Projektionen von weiblichen Eigenschaften darstellen, die als zu sich selber gehörig akzeptiert werden, wogegen sich allerdings meist heftige Widerstände erheben. ${ }^{9}$

Die junge Beatrice ist die Seelenführerin (wie die gleichnamige Frauengestalt in Dante Alighieris Divina Commedia), die Motivation für Emil, sich wieder der hellen Welt zu widmen. Die Mutter Demians, Frau Eva, ist eine andere Art von Anima. Sie ist die Vereinigung von Mutter und Dämon, von Schicksal und Geliebter, sie verkörpert den Archetyp der ewigen Frau, die zugleich Gebärerin, Todbringende, Geliebte, Tochter und Schwester ist. Sie trägt selber das Kainszeichen auf der Stirn

8 Jung, Carl Gustav: Aion - Beiträge zur Symbolik des Selbst. [Gesammelte Werke, Bd. 9/ 2]. Ostfildern: Patmos Verlag 2001. S. 423.

9 Vgl. auch: Jacobi, Jolande: Der Weg zur Individuation. Olten: Walter, 1971, S. 58. 
und weist - wie ihr Sohn - androgyne Züge auf; sie erscheint als weder jung noch alt, weder gut noch böse. Sie besitzt alle Eigenschaften von Demian, jedoch in einer wesentlich höheren Intensität, und repräsentiert genau das Ziel, nach dem Sinclair strebt. Sie scheint auch oft eher ein Teil von Sinclairs Unterbewusstsein zu sein als eine real existierende Person.

Alle diese Archetypen haben ihre bestimmte Rolle bei der Beeinflussung des jungen Emil, der seine eigene Identität zu finden hat. Die letzten zwei Archetypen, nämlich der Schatten und die Anima sind auch in Urtrost von Tamási zu identifizieren. Der Schatten taucht in der Gestalt des Schriftführers auf, der bösartig den Bruder des Toten als Verdächtigen darzustellen versucht. Er interpretiert alle Handlungen von Ádám derart, als wären diese gegen das Gesetz, gegen die christliche Religion und gegen seinen eigenen Bruder durchgeführt worden.

Die Frauenfigur als Archetyp von Anima hat auch in Urtrost eine Schlüsselrolle. Jula Kispál, eine Waise, die Adoptivtochter des Freundes von Csorja, stellt ebenfalls eine Figur dar, die weder Kind noch Frau, weder gut noch böse, und zugleich Opfer und Täterin, Lebensspendende und Todbringende ist. Ádám träumt von ihr und sieht in ihr den Trost für die Zukunft, die Hoffnung: „Du Trost, du Hoffnung, du warmes Goldhaus“"10 wird sie genannt, aber auch Bence Gálfi, der Gendarm ist in sie verliebt und jagt sie in seinen Träumen. In keinem der Texte wird die Anima jedoch erreicht, sowohl Frau Eva, Beatrice als auch Jula bleiben nur Traum, Hoffnung und Sehnsucht. „Jula, Jula, liebe Kindfrau, warte, ich gehe, ich gehe jetzt zu dir [...] ich werde mich durch dich neu erschaffen. "11 - bricht es aus Csorja heraus, als er sich Rechenschaft gibt, dass er alles, was er liebt, hinter sich lassen und flüchten muss. Er sieht in Jula

${ }^{10}$ „te vigasztalás, te remény, te meleg aranyház“. [Übersetzung der Verfasserin].

${ }^{11}$ „Jula, Jula, drága gyermekasszony, várj, megyek, most hozzád megyek, s el nem bujdosom, míg általad újra nem teremtem magamot”. [Übersetzung der Verfasserin]. 
die Hoffnung und das Versprechen eines neuen Lebens, die ihn und seinen Stamm in die Zukunft hinüberretten können. Jula steht auch zwischen zwei Welten: Einerseits möchte sie sich in die geschlossene Gesellschaft des Dorfes integrieren und sich nach den Lehren des Christentums sittlich, fromm und ernst verhalten, fühlt sich jedoch zugleich zur Wildheit der archaischen Welt und dem starken Mann hingezogen, der über sich selbst sagt: „Ich bin der ewige Funke, der aus der Macht von Attila geblieben ist." ${ }^{12}$ Sie ist schließlich auch diejenige, für die Csorja sterben wird: Bence Gálfi erschießt ihn aus Eifersucht. „Gott, Gott, wahrer Gott!“ schreit am Ende des Dramas Jula, als sie den ermordeten Ádám Csorja findet. Daraufhin antwortet ihr Pflegevater resigniert: „Unser Urblut ist vertrocknet“ und sie umarmen sich erschrocken und beginnen langsam und dann allmählich immer wilder zu tanzen. Dieser Tanz erinnert an die alten Schamanentänze der heidnischer Kultriten, eine Umarmung der Liebe mit dem Tod, ein Versuch, das Gleichgewicht der Welt wiederherzustellen; nach dem Verlust bzw. der Transformation der althergebrachten Werte eine neue Ordnung zu erschaffen. Damit symbolisiert Urtrost die Lösung, die aus dem tragischen Zustand herausführt: die Versöhnung der alten Moral mit dem Neuen.

Eine andere Gemeinsamkeit zwischen den Gestalten der beiden Texte ist die Verkörperung des „Urwissens“ in einer Figur. Bei Hesse ist es die Figur des Demian, bei Tamási die Figur des Ambrus, die eine Art Urwissen verkörpern. Über Ambrus wird gesagt: „Urwissen saß auf seinem Gesicht" ${ }^{\text {"13 }}$ und über Demian erfahren wir: „Denn ich sah in seinem Blick wieder diese seltsame, tierhafte Zeitlosigkeit, dies unausdenkliche Alter." und:

Ich sehe ihn zur Schule gehen [...] fremdartig, einsam und still, wie gestirnhaft $[\ldots]$ von einer eigenen Luft umgeben, unter eigenen

${ }^{12}$ „én vagyok az Attila hatalmából megmaradt örök szikra”. [Übersetzung der Verfasserin].

13 „Ösértelem az arcán” [Übersetzung der Verfasserin]. 
Gesetzen lebend [...] mit wissenden Augen [...]. Ich sah Demians Gesicht und ich sah nicht nur, daß er kein Knabengesicht hatte, sondern das eines Mannes, ich sah noch mehr [...]. Es war, als sei auch etwas von einem Frauengesicht darin, und namentlich schien dies Gesicht mir, für einen Augenblick, nicht männlich oder kindlich, nicht alt oder jung, sondern irgendwie tausendjährig, irgendwie zeitlos, von anderen Zeitläuften gestempelt, als wir sie leben.

Weitere Elemente, die ebenfalls einen Bezug zu Jung und seinen Theorien haben, sind die Träume. In beiden Werken spielen die Träume eine sehr wichtige Rolle, und werden als Prophezeiung interpretiert. Emils Traum vom Sperber sagt seine Zukunft vorher: „Der Vogel kämpft sich aus dem Ei. Das Ei ist die Welt. Wer geboren werden will, muss eine Welt zerstören."

Der Vogel, der sich aus einem Ei kämpft, symbolisiert Emil und seinen Individuationsweg. Genau wie der Vogel so muss auch Emil den Schutz um sich aufbrechen um neu geboren werden zu können. Und Bence Gálfi hat ebenfalls einen Traum von einer Fee mit rotem Gewand, deren Haar schwarze Seide ist und die sich schließlich als die liebe- und todbringende Jula entpuppt: Zerstörung und Neugeburt, Tod und Leben verschmelzen in diesem Traum ebenfalls, die Sehnsucht nach der Liebe von Jula und die Vorahnung des Mordes an Ádám.

Ein letzter gemeinsamer Punkt in den beiden Texten ist die historische Einbettung bzw. die damit verknüpfte Änderung der Welt. Bei Hesse ist der Krieg der Wendepunkt, der Änderungen mit sich bringt und bei Tamási die Jahrhundertwende. Das Drama von Tamási endet mit dem ersten Tag des 20. Jahrhunderts und ähnlich wie bei Hesse, verkündet dieser Morgen Erneuerung und Hoffnung für die Szekler. Er fordert sie auf und ermutigt sein Volk zum Weiterkämpfen und zur Bewahrung der Hoffnung, dass bessere Tagen kommen werden.

Das dramatische Werk von Tamási kann als eine verstörende, zeitlose Ballade über die Grundfragen der menschlichen Existenz gelesen werden. Der volkstümliche Balladenton ist 
mit der heidnisch-christlichen Mythologie verflochten: Urtrost rettet Erinnerungen und Stimmungen aus einer Zeit herüber, in der es noch keine scharfe Grenze zwischen heidnischen und christlichen Riten gab. Mithilfe des Konstruktionsprinzips des antiken Dramas und des mittelalterlichen Mysterienspiels und mit der Mischung des völkischen Bauerndramas mit den innovativen Lösungen des Avantgarde-Theaters ist es Tamási gelungen, mit diesem Stück neue Wege in der ungarischen Literatur einzuschlagen - obwohl dessen Rezeption lange auf sich warten ließ: 1924 geschrieben, wurde es erst 1973 veröffentlicht.

Wichtig ist es auch, unsere Aufmerksamkeit auf den Titel des Dramas von Tamási zu lenken: Hier erscheint das von den Autoren des Expressionismus häufig verwendete Präfix , ur-“"14, das kontextbedingt sowohl auf einen Ausgangszustand, auf den Ursprung, auf das vor langer Zeit Existierende (z.B. Urmensch), aufdas Unverfälschte/ die Echtheit und aufdie Unverbrauchtheit des im Wortstamm Genannten (z.B. Urkraft) hinweist, als auch die im Basissubstantiv Genannten vorhergehende oder nachfolgende Generation kennzeichnet (z.B. Urgroßvater, Urenkel).

Zusammenfassend lässt sich feststellen, dass es mit Zuhilfenahme der an C.G. Jung angelehnten Archetypenlehre bzw. des Konzepts des kollektiven Unbewussten den Autoren beider behandelten Werke gelingt, die Grundfragen des menschlichen Seins, die von Urkräften regiert und tief verankert im Gedächtnis der Menschheit sind, adäquat und spannend darzustellen, bzw. die tiefenpsychologisch fundierte literarische Tradition um zwei herausragende Werke zu bereichern.

${ }^{14}$ Man denke z.B. an das Gedicht Urtod von August Stramm. 


\section{Literaturverzeichnis:}

\section{Primärliteratur}

Hesse, Hermann: Demian. Die Geschichte von Emil Sinclairs Jugend. Berlin: Fischer 1920.

Tamási, Áron: Ösvigasztalás. In: Tiszatáj 27 (1973), H.5, S. 6-25, bzw. Tiszatáj 27 (1973), H.6, S. 23-48.

Jung, Carl Gustav: Die Archetypen und das kollektive Unbewußte. [Gesammelte Werke Bd. 9/1], Olten: Walter 1976.

Jung, Carl Gustav: Aion - Beiträge zur Symbolik des Selbst. [Gesammelte Werke, Bd. 9/ 2]. Ostfildern: Patmos Verlag 2001.

Jung, Carl Gustav: Die Struktur der Seele. In: ders.: Die Dynamik des Unbewussten. [Gesammelte Werke, Bd. 8], Ostfildern: Patmos Verlag 1995.

\section{Sekundärliteratur}

Baumann, Günter: Hesses „Demian“ im Lichte der Psychoanalyse C.G. Jungs. In: Materialien zu Hermann Hesses „Demian“. Zweiter Band. Hrsg. Von Volker Michels. Frankfurt am Main: Suhrkamp 1997.

Ball, Hugo: Hermann Hesse: Sein Leben und sein Werk. Oberursel: Gröls Verlag [Edition Werke der Weltliteratur] 2019.

Egbers, Marcel: Hesses Demian. Über Mythen, Religion und Esoterik zur Selbstfindung. München: GRIN Verlag 2001. Jacobi, Jolande: Der Weg zur Individuation. Olten: Walter, 1971. 